\title{
Vibration Analysis of an Aero-Engine Compressor Blade
}

\author{
*Kai Zhang \\ Department of Mechanical Engineering \\ and Automation, Northeastern \\ University, Shenyang, China, 110819 ,* \\ 610714235@qq.com
}

\author{
Wenhao Qu \\ Department of Mechanical Engineering \\ and Automation, Northeastern \\ University, \\ Shenyang, China, 110819
}

\author{
Wanshan Wang \\ Department of Mechanical Engineering \\ and Automation, Northeastern \\ University, \\ Shenyang, China, 110819
}

\begin{abstract}
A detailed three-dimensional finite element analysis model for an aero-engine compressor blade was established. Blade vibration response was experimented, considering a dry friction non-linear response method for solving for the fan blades. It can't be calculated using the ANSYS platform when considering the dry friction situation because there is a strong nonlinear shroud contact surfaces. This article uses the trajectory tracking and time-frequency transformation method to solve the fan blades with dry friction and nonlinear response, and determine the size of the shoulder contact surface speed, pressure and contact stiffness. Then we get the frequency response of the fan blades of the state curve and the displacement, stress contours after calculating. The comparison of the results by response displacement under the condition of all the coordination with two pairs of nodes to take into account dry friction, vibration significantly reduced, but the displacement and stress distribution changed little, due to the presence of friction damping. The calculation process is quite complicated, but more precise calculations when considering dry friction damper with dry friction and nonlinear response to the solver calculation.
\end{abstract}

Key words-Aero-engine; compressor blade; Non-linear response; Dry friction

\section{INTRODUCTIONS}

The blade is one of the main parts of aircraft engine, in the work plays the role of energy conversion, often called the heart of the engine [1]. Blade working environment is bad, in addition to withstand high speed rotating aerodynamic force, centrifugal force and vibration load outside, but also by the effect of thermal stress. Compressor blade number and sizes, the engine in the big speed work a blade centrifugal force [2]. If there is a crack when prone to fracture, because of the vibration of the blade to high cycle fatigue problem is particularly common, complex, and accounts for about $25 \%$ of the total accidents. Therefore, it is in the blade design process to establish appropriate finite element model and static strength and vibration modal and response analysis has very important practical significance.

Compressor blade between two convex shoulder contact with contact stiffness between the direct impact on the blade response of the resonance frequency and damping effect is good or bad, contact stiffness of accurate or not directly affect the nonlinear response calculation to the accuracy of the results [3]. Currently speaking, contact stiffness mostly according to experimental methods or experience to a given, and this is limited to some special situation, can't use, and gave to solve nonlinear response brought a lot of inconvenience [4]. This paper puts forward a kind of make use of the finite element method to solve contact stiffness method, can reduce the use of test method determine the tangential contact stiffness need a lot of time and money.

\section{CONTACT RIGIDITY AND CALCULATION METHOD TO DESIGN}

A engine compressor blade using convex shoulder structure reduce vibration of the blade by mesh division, the division after completion of the finite element model is shown as shown in figure 1.1. The 20 node hexahedron mesh, total is divided into 2753 units and 15694 nodes.

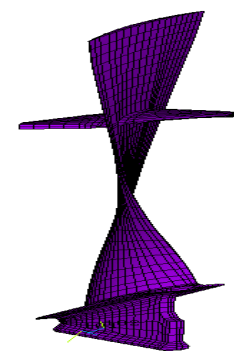

Fig.1.1 Blade finite element model

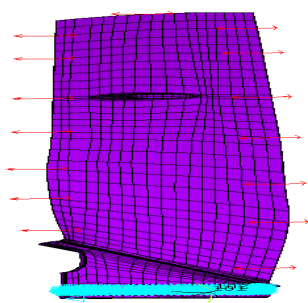

Fig.1.2 Leaves suffered a steady-state excitation force

Blade by aerodynamic force is more complex, cause the vibration of the blade vibration force also is very complicated, it is difficult to accurately describe is adopted in this paper, a simplified steady-state vibration force, axial (X axis direction) loading $2 \mathrm{n}$ steady-state vibration force, circumferential (Y-axis direction) loading $3 \mathrm{n}$ steady-state vibration force. In figure.1.2 shows the loading steady-state 
vibration force.

This paper mainly analyzes the contact surface shape, positive pressure $\mathrm{FN}$ and friction factor $\mu$ to contact the influence law of stiffness. In finite element method to solve the stiffness is used in the static elastic contact analysis, the contact pressure and temperature is constant. So in the tangential stiffness analysis will contact surface friction coefficient $\mu$ as constant.

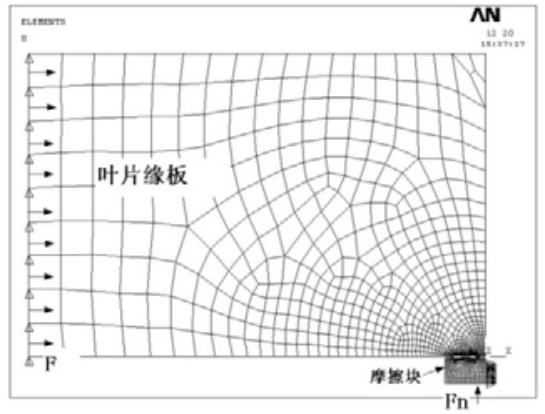

Fig.2 Calculate the finite element model of the tangential stiffness

For tangential contact stiffness calculation of finite element model as shown in figure. 2 shows, in order to avoid friction block chamfering place appear stress concentration phenomenon, friction block and leaf margin plate of the contact area for arc surface. Friction block on the right side of the node $\mathrm{X}$ direction completely constraints, blade edge panel on the left of the node $\mathrm{Y}$ direction completely constraints. Friction block in the positive pressure under the action of Fn and leaf margin plate contact, and then to leaf margin plate applied force $F$, when $F$ is greater than the positive pressure due to the friction produced when blade flange plate and the friction between block started to slide.

\section{BLADE TANGENTIAL STIFFNESS CALCULATION}

This part calculates coefficient of tangential contact stiffness by using the design method for different positive pressure and different friction. In the friction piece of $10 \mathrm{n}$ on the positive pressure, different friction coefficient corresponding calculation results can get force and slip displacement relationship curve, shown in figure.3.

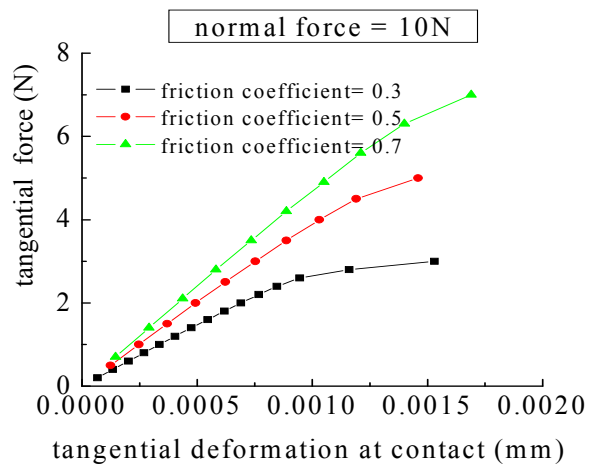

Fig.3.1 The relationship between the force and the contact point cut in the deformation when positive pressure is $10 \mathrm{~N}$
In figure.3.1, three line linear segment corresponding slope is the friction coefficient of $10 \mathrm{n}$ positive pressure under the action of tangential contact stiffness. From figure.3.1 can know, friction coefficient is 0.3 its tangential contact stiffness for $2930 \mathrm{n} / \mathrm{mm}$, friction coefficient is 0.5 tangential stiffness for $3990 \mathrm{n} / \mathrm{mm}$, friction coefficient is 0.7 tangential stiffness for $4730 \mathrm{n} / \mathrm{mm}$. Use the same method can get different positive pressure, different friction coefficient under the result of the contact stiffness.

Use the same method to get the rest of the different positive pressure under the action of tangential force and the contact point tangential deformation curve. In figure. 3 to every line take linear segment of slope can be different under positive pressure, the coefficient of friction corresponding tangential contact stiffness, as is shown in table.1.

TABLE.1 RADIUS OF 50.6MM WHEN DIFFERENT POSITIVE PRESSURE COEFFICIENT OF FRICTION CORRESPONDING CUT TO THE CONTACT STIFFNESS

\begin{tabular}{cccc}
\hline $\begin{array}{c}\text { Positive } \\
\text { pressure } \\
(\mathbf{N})\end{array}$ & $\begin{array}{c}\text { the tangential } \\
\text { contact } \\
\text { stiffness when } \\
\mathbf{u = 3 . 0}(\mathbf{N} / \mathbf{m m})\end{array}$ & $\begin{array}{c}\text { the tangential } \\
\text { contact stiffness } \\
\text { when } \boldsymbol{\mu}=\mathbf{0 . 5} \\
(\mathbf{N} / \mathbf{m m})\end{array}$ & $\begin{array}{c}\text { the tangential } \\
\text { contact } \\
\text { stiffness when } \\
\boldsymbol{\mu = 0 . 7}(\mathbf{N} / \mathbf{m m})\end{array}$ \\
\hline 10 & 2930 & 3990 & 4730 \\
20 & 3350 & 4370 & 4966 \\
50 & 4761 & 5702 & 6250 \\
100 & 6770 & 7723 & 8227 \\
150 & 7660 & 8442 & 8842 \\
200 & 8197 & 8873 & 9211 \\
400 & 9350 & 9805 & 10001 \\
800 & 10272 & 10551 & 10691 \\
1600 & 11066 & 11208 & 11261 \\
\hline
\end{tabular}

For compressor blade convex shoulder contact surface, in the role of vibration in the above positive pressure along with the change of contact state can produce change, the corresponding stiffness value will also change [5]. To solve the get tangential contact stiffness with positive pressure change curve fitting, the index form of relation, as shown in figure 4 shows the friction coefficient is 0.3 fitting results. In the nonlinear response solving process, contact stiffness can make use of the positive pressure value through the expression get.

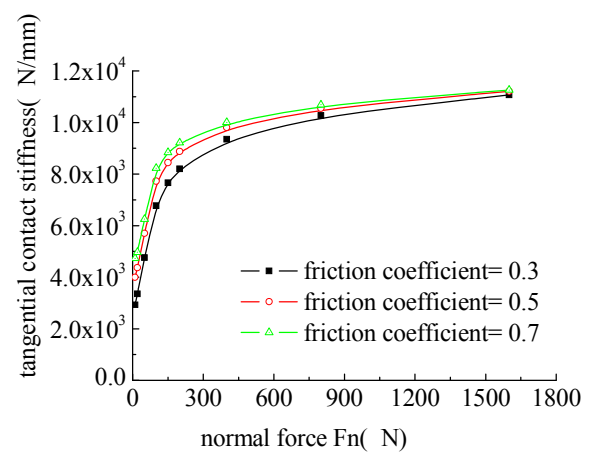

Fig.3.2 Tangential contact stiffness with positive pressure change in the curve 


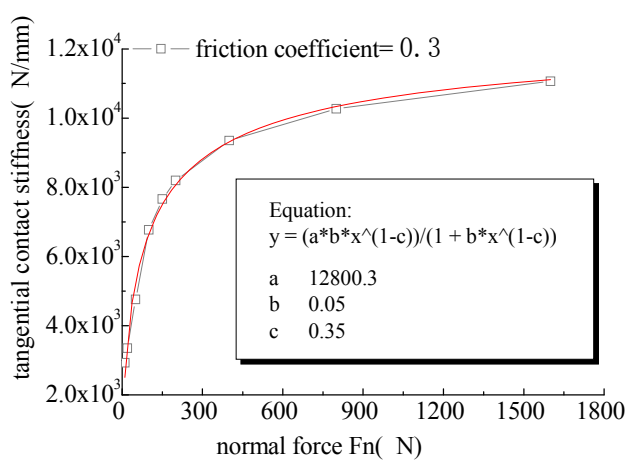

Fig.3.3 Coefficient of friction of 0.3 cut stiffness with positive pressure changes in the fitted curve

\section{SOLVING}

In the work speed within the scope of the nonlinear response analysis, the vibration frequency for speed 10 times frequency [6]. For different speed, the contact surface initial positive pressure value can get according to the calculation results interpolation. Contact the initial positive pressure value is more than $2000 \mathrm{~N}$, according to the fitting results, contact stiffness changes will be very small, so the analysis does not consider contact stiffness along with the speed of change, at a constant value: tangential stiffness is $0.6 \mathrm{E} 4$ states $\mathrm{N} / \mathrm{mm}$, normal stiffness for $1 \mathrm{E} 4 \mathrm{n} / \mathrm{mm}$.

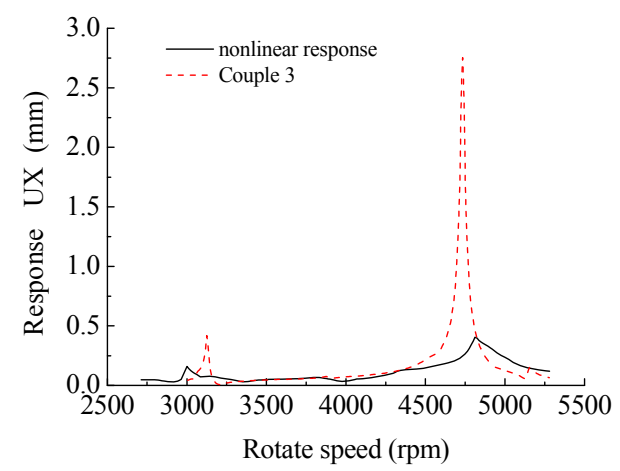

(a) $\mathrm{X}$ direction displacement response curve with engine speed

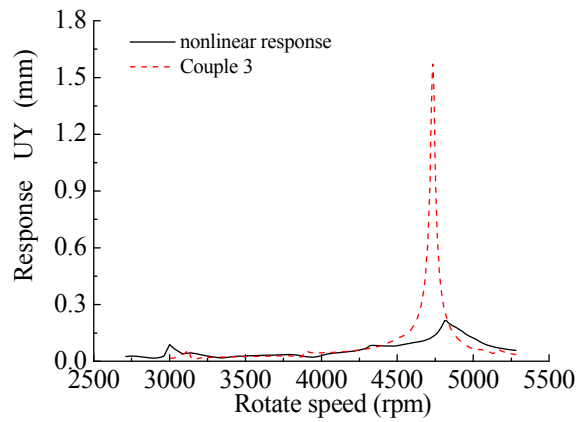

(b) Y direction displacement response curve with engine speed

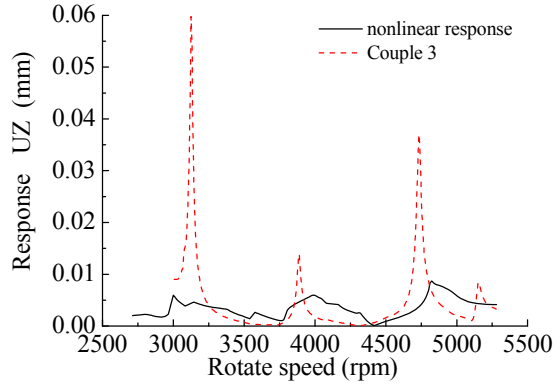

(c) $\mathrm{Z}$ direction displacement response curve with engine speed

Fig.4.1 Node 3133 of the displacement response curve with engine speed

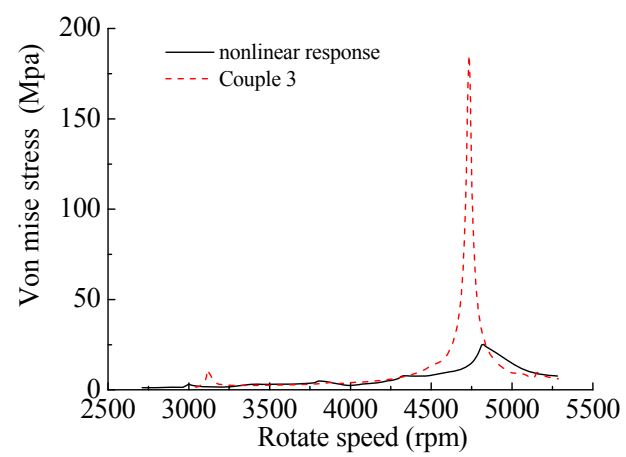

(a) Equivalent stress curve with engine speed

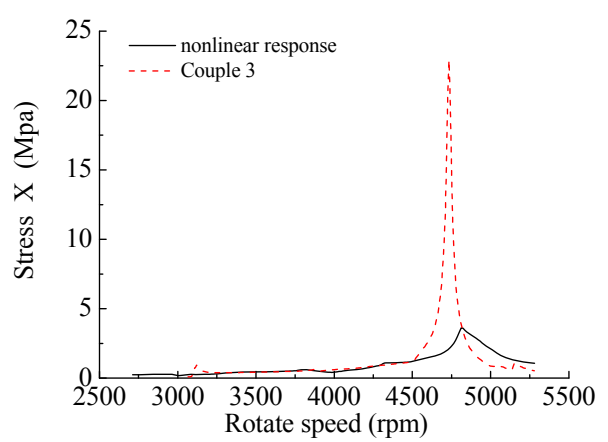

(b) $\mathrm{X}$ direction stress curve with engine speed

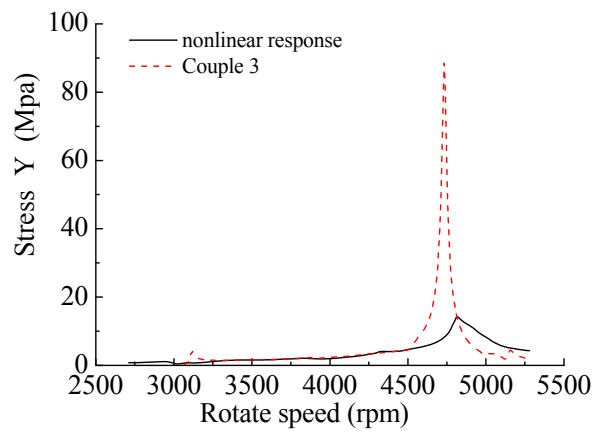

(c) Y direction stress curve with engine speed 


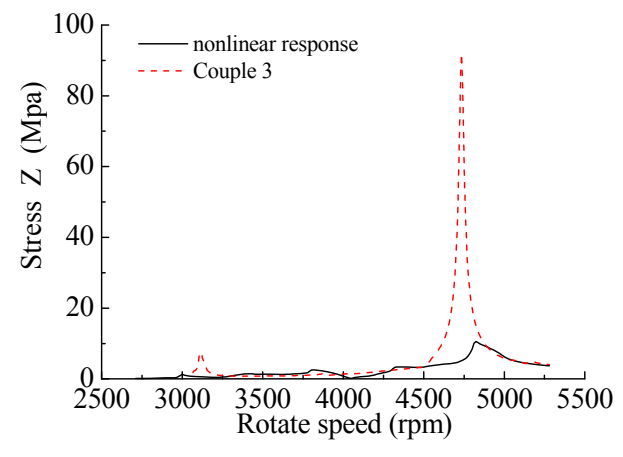

(d) $\mathrm{Z}$ direction stress curve with engine speed

Fig.4.2 Node 3370 of the stress response curve with engine speed

From the figure.4.1 and figure.4.2 can be seen in the existing compressor blade convex shoulder structure dry friction damping effect is very obvious, in the whole speed range of each direction vibration displacement and stress has a lot of lower. Displacement maximum response by coordination of $2.75 \mathrm{~mm}$ (4700 rpm) be reduced to consider dry friction damping effect of $0.43 \mathrm{~mm}(4800 \mathrm{rpm})$; The maximum equivalent stress by coordination of $184 \mathrm{Mpa}$ (4700 rpm) reduced to consider dry friction damping effect $24 \mathrm{Mpa}(4800 \mathrm{rpm})$.

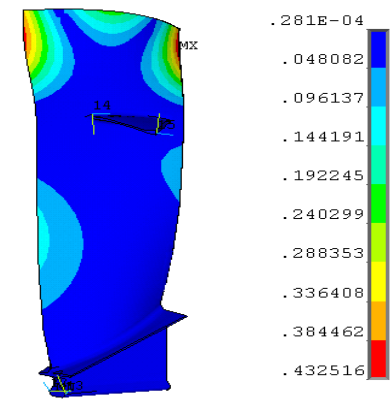

(a) Displacement response images

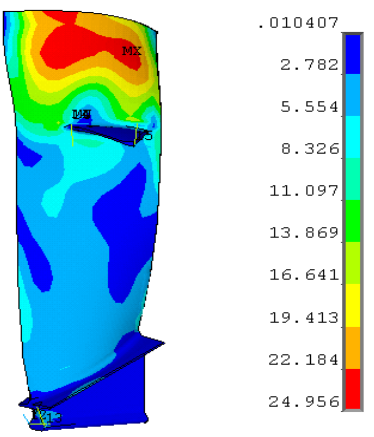

(b) the stress response cloud images

Fig.4.3 Consider the maximum response of the fan blades cloud images in the operating speed range of design state dry friction damping

Calculate the stress response for different frequency blade under stress and displacement response of the distribution by finite element method. Consider dry friction damping action get work speed within the scope of the maximum response vibration mode, and don't take time two node displacements coordinate the entire sixth order resonance vibration mode similar, but the response amplitude greatly reduced. Maximum stress response distribution and does not consider the dry friction stress distribution is also similar, but stress amplitude greatly reduced.

\section{CONCLUSIONS}

1) Different friction coefficient corresponding tangential contact stiffness with positive pressure change curve. Contact surface tangential stiffness with normal positive pressure increases while the, but the increase rate tends to slow. Friction coefficients on the tangential stiffness influence is mainly embodied in the positive pressure is less than $400 \mathrm{n}$, when positive pressure is large friction coefficient of the response is very small.

2) In this paper research compressor blade speaking. Through calculation, the actual engine work process contact only area of contact and pressure distribution is not uniform. So according to the above analysis, we can know, for the compressor blade speaking, the contact stiffness value influenced by the contact surface shape and the influence of the friction coefficient is very small, and working process of contact stiffness should be changed little.

3) Convex shoulder structure of different contact surface state will not only affect the blade resonance peak size, also will change resonance speed. Therefore, when the design to comprehensive consideration of the damping effect and resonance speed, best can directly to avoid harmful resonance; If avoided, should make vibration stress reduced to a reasonable level.

\section{REFERENCES}

[1] Weiqiang Zhao, Yongxian Liu and Mowu Lu, "FEA on vibration characteristics analysis of an aero-engine compressor blade," IEEE Control Syst.Soc. China, vol. C7440, pp. 3248-50, May 2012.

[2] Bing Chen, Ting Yang and Tao Feng, "Ontology-based aero-engine compressor blade manufacturing quality analyzing," IEEE Wuhan Sect. China, vol C7480, pp.4, Dec 2009.

[3] Hammouda, M.M.I and Pasha, R.A, "Modelling of cracking sites/decelopment in axial dovetail joints of aero-engine compressor discs," International Journal of Fatigue. Vol 29, pp.30-48, January 2007.

[4] Weiqiang Zhao, Yongxian Liu and Mowu Lu, "Vibration analysis of aero-engine compressor blade-disc coupling system," Appl.Mech.Mater. Vol 16-19, pp.264-268, 2009.

[5] Savkov,K, Doroshko,S and Urbaha,M, "Gas turbine aero engine compressor blade erosion and heat resistant coatings," Transp.Means-Proc.Int.Conf. pp.274-278, October 2011.

[6] Wei-Feng He, Ying-Hong Li and Wei Li, "Laser shock peening on vibration fatigue behavior of compressor blade," Hongkong Dongli Xuebao. Vol 26, pp.1551-1556, July 2011. 